\title{
Superficial Parotidectomy and Postoperative Drainage
}

Phillip J. Mofle, MD and Andrew C. Urquhart, MD

\begin{abstract}
Objectives: A shift toward shorter hospitalizations and outpatient procedures has become the standard in perioperative care. Two factors affecting the length of hospitalization following parotidectomy are duration of postoperative drainage and the use of surgical drains. Identifying factors that are predictive of postoperative drainage may allow earlier discharge or selection of patients suitable for outpatient procedures. The aim of this study was to identify any factors that may be predictors of postoperative drainage.
\end{abstract}

Design: Retrospective review.

Setting: A 500+ bed tertiary care medical center in central Wisconsin.

Participants: Patients who underwent superficial parotidectomies over a 5-year period.

Methods: Clinical charts were retrospectively reviewed. Age, gender, anticoagulation use, history of hypertension, estimated intraoperative blood loss, postoperative complications, total postoperative drainage, length of hospital stay, and final pathology were recorded for each patient. Spearman rank correlation was used to evaluate associations, and the Kruskal-Wallis test was used for subgroup comparisons.

Results: Ninety-six superficial parotidectomies were performed during the 5-year time period and 69 met our criteria for inclusion in the study. Final pathology was directly associated with postoperative drainage with benign tumors having significantly less drainage than malignant tumors $(P=0.0 \mathrm{I} I)$. Length of hospital stay was also significantly associated with postoperative drainage $(r=0.36$, $P=0.002)$. No significant associations with age $(P=0.209)$, gender $(P=0.904)$, history of hypertension $(P=0.780)$, or estimated intraoperative blood loss $(P=0.109)$ were noted.

Conclusions: Malignant pathology is associated with increased postoperative drainage and increased length of hospitalization. Accurately predicting malignancies preoperatively may expedite and facilitate postoperative planning and offer insight into the expected duration of postoperative drainage.

Keywords: Drainage; Health care costs; Length of stay; Parotid gland; Surgery

Reprint Requests: Andrew C. Urquhart, MD, Department of Otolaryngology-Head and Neck Surgery, Marshfield Clinic, 1000 North Oak Avenue, Marshfield, WI 54449; Tel: 7I5-387-527I; Fax: 7I5-389-3808; Email: urquhart.andrew@marshfieldclinic.org.
Received: December 21, 2007

Revised: April 25, 2008

Accepted: April 30, 2008

doi: $10.3121 / \mathrm{cmr} .2008 .787$ 
Table 1. Patient data.

\begin{tabular}{lllllll}
\hline & N & Mean & SD & Minimum & Median & Maximum \\
\hline Age & 69 & 56.9 & 15.9 & 24.0 & 58.0 & 88.0 \\
Total drainage (cc) & 69 & 49.2 & 45.7 & 0.0 & 35.0 & 260.0 \\
Estimated blood loss & 62 & 50.3 & 45.0 & 10.0 & 30.0 & 300.0 \\
Hospital days & 69 & 1.9 & 0.7 & 1.0 & 2.0 & 3.0 \\
\hline
\end{tabular}

$\mathrm{H}$ increased efficiency. Accordingly, a shift toward shorter hospitalizations and outpatient procedures has become the standard in perioperative care. ${ }^{1-6}$ A major factor affecting the length of hospitalization following parotidectomy remains the use of surgical drains. Drains obliterate dead space and approximate flaps to promote wound healing. ${ }^{7}$ By approximating the skin flaps, accumulation of blood, serous fluid, and saliva is avoided. Drains themselves are not a definite reason to keep a patient hospitalized. However, if a patient is sent home with a drain in place, there are many nursing and care issues related to the drain which may require further outpatient visits. In our facility, we keep the drains in place with the patient hospitalized until the total drainage over an 8-hour shift is less than $15 \mathrm{cc}$.

While drains may be considered standard postoperative care, little has been written in regard to the amount or duration of drainage. Identifying factors that are predictive of postoperative drainage may allow earlier discharge or selection of patients suitable for outpatient procedures. We show that there is a direct correlation between the final pathology, length of hospital stay, and total postoperative drainage.

\section{Methods}

Prior to data collection, the institutional review board of Marshfield Clinic/Saint Joseph's Hospital granted approval for a chart review of parotidectomies performed from October 1999 through September 2004. A total of 96 patients who underwent parotidectomies were identified. In an attempt to reduce the number of confounding variables, we focused our analyses on only those patients who underwent superficial parotidectomies without neck dissections. Thus, patients who underwent total parotidectomies or those with neck dissections were excluded from the final analyses. Of the 96 patients identified, 69 presented with a parotid mass within the superficial lobe and underwent a superficial parotidectomy and facial nerve dissection with no neck dissections. These 69 patients were included in the final analyses.

The surgical technique and extent of surgery for parotidectomy was similar in all cases. Postoperative drainage was recorded in the nursing records every 8 hours and as total drainage upon removal of the drain. A closed vacuum drainage system was used in all patients. Drains were placed to constant wall suction or mobile-vac at all times and were removed when drainage was less than $15 \mathrm{cc}$ over an 8-hour shift.

Patient data including age, gender, anticoagulation use, and history of hypertension were recorded. Additional variables in the analysis included estimated intraoperative blood loss, length of hospital stay, and final pathology.

Standard descriptive statistics were used to summarize the demographic and clinical characteristics of the study subjects and the surgical outcomes. The distributions of continuous measurements, including total drainage, were observed to be quite skewed. Accordingly, results were summarized with medians, as well as means, and were analyzed with nonparametric procedures. Spearman rank correlation was used to evaluate associations among continuous measures, and the Kruskal-Wallis test was used for subgroup comparisons.

\section{Results}

Sixty-nine superficial parotidectomies without neck dissection were performed from October 1999 through September 2004. The patients ranged in age from 24 to 88 years. Forty-six percent were male and $54 \%$ were female. Estimated intraoperative blood loss ranged from $10 \mathrm{ml}$ to 300 $\mathrm{ml}$ with a median of $30 \mathrm{ml}$. The total postoperative drainage ranged from $0 \mathrm{ml}$ to $260 \mathrm{ml}$ with a median of $35 \mathrm{ml}$, and the duration of hospitalization ranged from 1 to 3 days with a median of 2 days (table 1). The final pathology included benign tumor in $53(77 \%)$ patients, inflammatory disease in 7 $(10 \%)$ patients, and malignant tumor in $9(13 \%)$ patients (table 2).

Final pathology was shown to be significantly associated with postoperative drainage. Despite undergoing the same extent of surgery, benign tumors had significantly less drainage than

Table 2. Pathology of superficial parotidectomy cases

\begin{tabular}{lll}
\hline & Frequency & Percent \\
\hline Benign & 53 & 77 \\
Inflammatory & 7 & 10 \\
Malignant & 9 & 13 \\
\hline
\end{tabular}


Total Drainage by Pathology

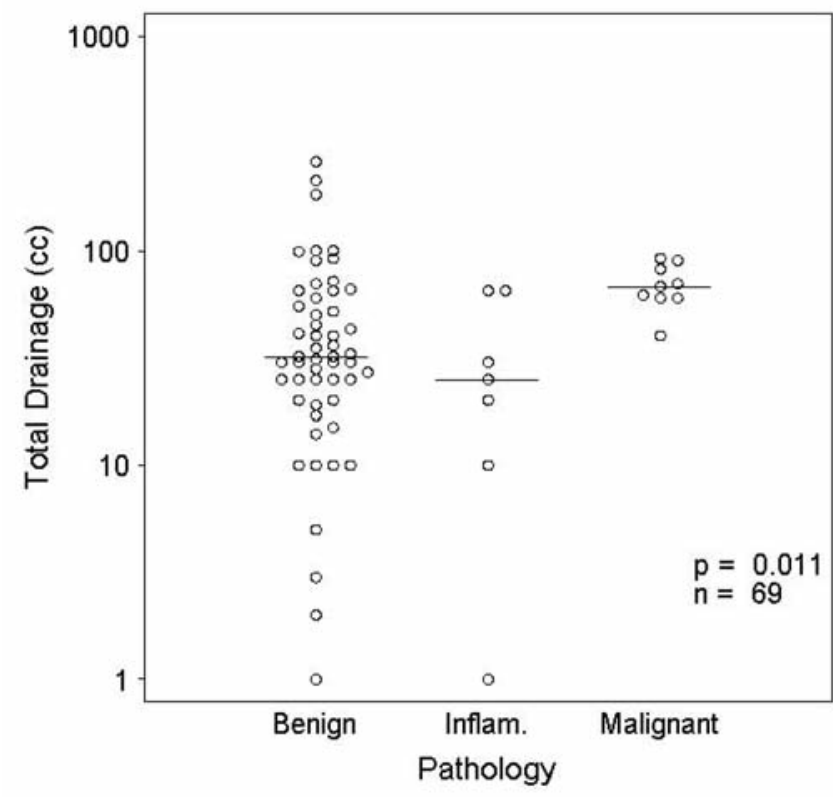

Figure 1. Total postoperative drainage (log scale) by pathology. Horizontal bars show the medians. The number of observations and the $P$-value from the Kruskal-Wallis test are shown in the lower right.

malignant tumors $(P=0.011)$ (figure 1). Length of hospital stay was also shown to be significantly associated with postoperative drainage $(\mathrm{r}=0.36, P=0.002)$ (figure 2). This finding was expected given that reduction of drainage to less than $15 \mathrm{ml}$ over an 8-hour shift is required for discharge at our facility. No significant associations of drainage with age $(P=0.209)$, gender $(P=0.904)$, history of hypertension $(P=0.780)$, or estimated blood loss $(P=0.109)$ were noted. Only two of the 69 patients were on Coumadin, which was held 5 days prior to surgery and resumed after discharge. Given this small number, the relationship between Coumadin use and postoperative drainage could not be determined.

\section{Discussion}

Parotid tumors and their treatment have become increasingly common as our understanding of parotid anatomy and pathology has expanded and our diagnostic capabilities advanced. Similarly, there has been an evolution of techniques from enucleation or local resection to superficial or total parotidectomy based on pathology. As in most series, the majority of resections (77\%) were performed for benign disease. As the population ages, however, malignancy will no doubt continue to play a role in parotid disease. As with most head and neck operations, drainage of the operative site has been standard practice whether a superficial or total parotidectomy has been performed. While there appears to be general agreement concerning the use of drains, the literature contains very little information on the expected behavior of the drains or recommendations on their management. $4,5,8,9$
As medical costs continue to rise, alternative approaches that reduce expenses and are proven to be safe and effective become attractive. Shorter hospital stays and outpatient operations are increasingly popular for a growing number and variety of procedures. Mounting surgical experience and technological advances promise to maintain this trend in the foreseeable future. Parotidectomy is no exception in this regard, as reports of outpatient procedures document feasibility. ${ }^{10}$ Despite these advances, the care of drains postoperatively remains ill-defined. We sought to document the postoperative drainage after parotidectomy and identify factors that may be contributory.

Published series on parotid surgery vary in their indications for drain removal from volume in a given time period to simply removing the drain after 2 to 7 days. ${ }^{8} 9$ We routinely used less than $15 \mathrm{cc}$ drainage over 8 hours as our trigger for drain removal, unless clinical factors necessitated longer drainage periods. Our rates of seroma formation (2\%) and salivary fistula $(0 \%)$ compare favorably to published series suggesting adequate drainage duration. $.9,11-13$

Preoperative predictors of drainage after parotidectomy could be helpful in determining length of hospitalizations or potentially in selecting individuals amenable to outpatient procedures. Our study revealed no significant association of drainage with age, gender, history of hypertension, or anticoagulation use. Thus, these variables cannot be used to predict amount of postoperative drainage and associated length of hospitalization.

\section{Total Drainage vs. Length of Hospitalization}

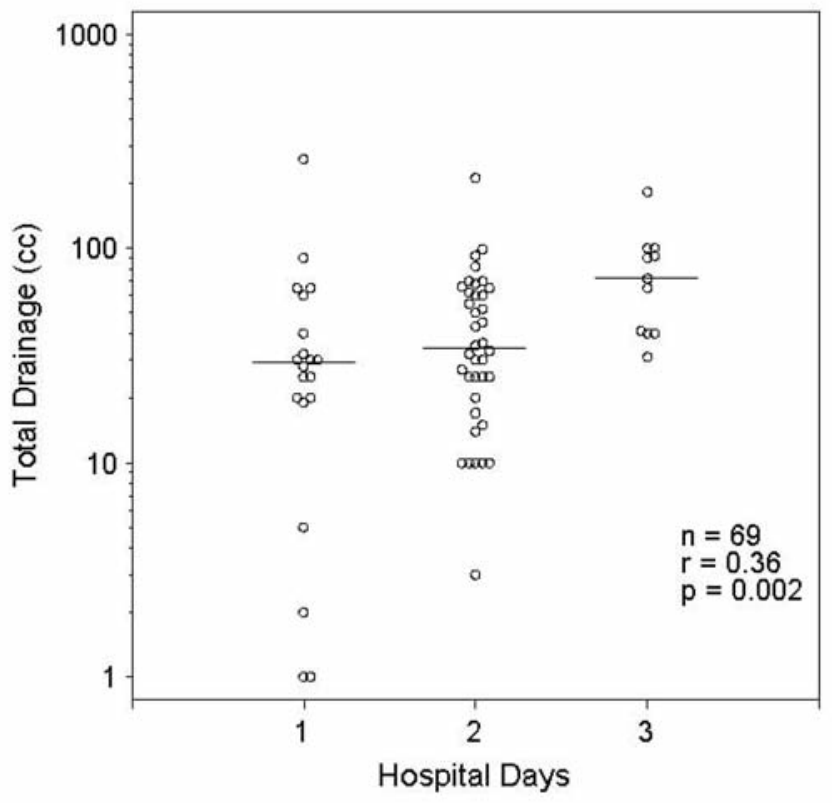

Figure 2. Total postoperative drainage (log scale) by length of hospitalization. The number of observations, the Spearman correlation, and associated $P$-value are shown in the lower right. Horizontal lines show the daily medians. 
While final pathology is not definitely known preoperatively, clinical factors with the inclusion of fine needle aspiration and computed tomography scans will often alert the clinician to cases that may have a higher risk of harboring malignancy. When a malignancy is suspected, a more complete resection is usually performed. A total parotidectomy and neck dissection is usually performed in high grade malignancies. With low grade malignancies, a superficial parotidectomy is usually adequate assuming gross clearance of all disease. We excluded total parotidectomies with combined neck dissection in the study, as the extent of surgery itself would obviously increase the amount of drainage. Final pathologic classification of the lesions was found to be a significant predictor of total postoperative drainage. Although the extent of surgery was the same for malignant and benign cases, total drainage was higher for malignant cases. Thus, results of preoperative diagnostic procedures may assist clinicians in predicting total postoperative drainage. If malignancy is suspected, one would expect greater total postoperative drainage and longer duration of hospitalization.

In our experience, postoperative drainage is the biggest factor in determining length of hospitalization following parotidectomy. Our referral base is almost entirely rural, and our patients commonly live considerable distances from the hospital and clinic. This presents a problem in the management of drains as an outpatient and further underscores the importance of predicting drainage. Further studies are needed to evaluate the process of prospectively predicting drainage after parotidectomy. As surgical care evolves and technological advances continue, the ability to select patients who are amenable to outpatient procedures or perhaps observation status postoperatively will become useful in our effort to reduce costs. With ambulatory surgery centers becoming commonplace and the variety of procedures performed broadening, parotidectomy appears poised to be included in the future of "day surgery."

\section{Conclusion}

Malignant pathology is associated with increased postoperative drainage and increased length of hospitalization following superficial parotidectomy. Although a total parotidectomy and neck dissection is usually performed for high grade malignancies, superficial parotidectomies are adequate for many low grade malignant tumors. Superficial parotidectomy is also performed for known or suspected metastatic cancer to the parotid lymph nodes. Accurately predicting malignancies preoperatively may expedite and facilitate postoperative planning and offer insight into the expected duration of postoperative drainage.

\section{Acknowledgments}

The authors thank Marshfield Clinic Research Foundation for its support through the assistance of Jennifer Hayes, Alice Stargardt and Linda Weis in the preparation of this manuscript and Richard Berg for statistical analysis and support.

\section{References}

1. Hecht AD. Creating greater efficiency in ambulatory surgery. J Clin Anesth 1995;7:581-584.

2. Jarrett PE. Day surgery - the future. Ann Chir Gynaecol 1995;84:379-383.

3. Millikan KW, Deziel DJ. The management of hernia. Considerations in cost effectiveness. Surg Clin North Am 1996;76:105-116.

4. Prasad A, Foley RJ. Day case laparoscopic cholecystectomy: a safe and cost effective procedure. Eur J Surg 1996; 162:43-46.

5. Deutsch N, Wu CL. Patient outcomes following ambulatory anesthesia. Anesthesiol Clin North America 2003; 21:403-415.

6. Marcinko DE, Hetico HR. Economic outcomes analysis from an ambulatory surgical center. J Foot Ankle Surg 1996; 35:544-549.

7. Byers RM, Ballantyne AJ, Goepfert H, Guillamondegui OM, Larson DL, Medina J. Clinical effects of closed suction drainage on wound healing in patients with head and neck cancer. Arch Otolaryngol 1982;108:723-726.

8. Sinha UK, Ng M. Surgery of the salivary glands. Otolaryngol Clin North Am 1999;32:887-906.

9. Henry LR, Ridge JA. Parotidectomy. ACS Surgery: Principles and Practice. Available at: http://www.acssurgery.com/cgi-bin/publiccgi.pl?loginOP. Accessed August 17, 2007.

10. Odell MJ, Durham JS. Parotid surgery in an outpatient setting: the Vancouver Hospital experience. J Otolaryngol 2003;32:298-301.

11. Bova R, Saylor A, Coman WB. Parotidectomy: review of treatment and outcomes. ANZ J Surg 2004;74:563-568.

12. Helmus C. Subtotal parotidectomy: a 10-year review (1985 to 1994). Laryngoscope 1997;107:1024-1027.

13. Wax M, Tarshis L. Post-parotidectomy fistula. J Otolaryngol 1991;20:10-13.

\section{Author Affiliations}

Phillip J. Mofle, MD

Department of General Surgery

Marshfield Clinic Indianhead Center

1020 Lakeshore Drive

Rice Lake, Wisconsin 54868

USA

Andrew C. Urquhart, MD

Department of Otolaryngology-Head and Neck Surgery

Marshfield Clinic

1000 North Oak Avenue

Marshfield, Wisconsin 54449

USA 